\title{
Anestesia para cirugía fetal: Consideraciones anestésicas y alcances terapéuticos
}

\section{Implications and anesthetic considerations for fetal surgery}

\author{
Carla Maldonado1, Pedro Torres², Daniel Riquelme³
}

\begin{abstract}
Fetal surgery is a field that has experienced great progress in recent decades. Advances in prenatal imaging techniques have allowed treatment of in-utero fetal pathologies during the prenatal period, so that it is currently possible to intervene in the natural history of certain alterations in the development of the fetus, avoiding sequelae in the newborn and in its subsequent development in extrauterine life. The perioperative management of fetal surgery requires a multidisciplinary team, constituting a challenge for the anesthesiologist to maintain the homeostasis of the mother and the fetus. The understanding of the maternal-fetal physiology together with an adequate management of the anesthetic techniques constitute the cornerstone for the success of the surgery.
\end{abstract}

\section{RESUMEN}

La cirugía fetal es un campo que ha experimentado gran progreso en las últimas décadas. El avance en las técnicas de diagnóstico prenatal por imágenes ha permitido tratamiento de patologías fetales in utero durante el período prenatal, de modo que actualmente es posible modificar la historia natural de determinadas alteraciones en el desarrollo del feto evitando secuelas en el recién nacido y en su posterior desarrollo en la vida extrauterina. El manejo perioperatorio de la cirugía fetal precisa de un equipo multidisciplinario, constituyendo un desafío para el anestesiólogo mantener la homeostasis de la madre y el feto. El entendimiento de la fisiología materno-fetal junto con un adecuado manejo de las técnicas anestésicas constituyen un factor fundamental para el éxito de la cirugía.

\section{Key words:}

Fetal anesthesia, fetal surgery, obstetric anesthesia

\section{Palabras clave:}

Anestesia fetal, cirugía fetal, anestesia obstétrica

\footnotetext{
Residente de Anestesiología.

Anestesiólogo.

Médico Cirujano.

Departamento Anestesiología y Medicina Perioperatoria, Hospital Clínico San Borja Arriarán, Universidad de Chile.
}

Fecha de recepción: 28 de marzo de 2019

Fecha de aceptación: 07 de abril de 2019

\section{ORCID}

https://orcid.org/0000-0002-1134-9369

Correspondencia:

Dra. Carla Maldonado

Email.carla.maldonado.benitez@gmail.com 


\section{Introducción}

E I Hospital Clínico San Borja-Arriarán ha sido pionero dentro de la Red de Salud para el desarrollo de la cirugía fetal. Desde el año 2015 a la fecha se han realizado 64 procedimientos fetales en este centro hospitalario. La indicación más frecuente ha sido la coagulación con láser en el síndrome de transfusión feto-fetal (STFF) con un $84 \%$ de los casos. También se han realizado otros procedimientos, como plastía de valvas uretrales posteriores y liberación de brida intraamniótica.

Los resultados a mediano y largo plazo aún son objeto de estudio por los ginecoobstetras maternofetales. Debido al desarrollo progresivo de estos procedimientos y la eventual implementación en otros hospitales, decidimos realizar una breve descripción de los casos de nuestro centro y revisión de la literatura.

\section{Tipos de cirugía fetal}

Las intervenciones fetales se pueden clasificar en tres categorías: Procedimientos mínimamente invasivos, cirugía fetal abierta y procedimientos EXIT (exútero intrapartum treatment) (Tabla 3).

Procedimientos mínimamente invasivos: Abarcan procedimientos con aguja guiados por ultrasonido e intervenciones fetoscópicas ecoguiadas. Los fetoscopios son insertados en la cavidad peritoneal donde se insufla dióxido de carbono de manera similar a la laparoscopía, y posteriormente, son introducidos en la cavidad uterina. Patologías como transfusión fetofetal en embarazos gemelares monocoriales, cierre de defecto de espina bífida, malformaciones urinarias y cardiacas, son susceptibles de ser tratadas mediante esta técnica[1]. Los procedimientos mínimamente invasivos más frecuentes se resumen en la Tabla 1.

Cirugías fetales abiertas: Se realizan mediante histerotomía, habitualmente durante las semanas 18 a 26 de gestación. Se exterioriza al feto total o parcialmente para corregir el defecto y, posteriormente, se devuelve a la cavidad uterina y se realiza la histerorra- fia[2]. La indicación más frecuente es la reparación de espina bífida, en el cual el cierre prenatal del defecto del tubo neural tiene mejor pronóstico neurológico que la reparación posnatal. Estudios de cohorte retrospectiva recientes han demostrado que la técnica abierta se asocia a mayores cambios hemodinámicos y mayores dosis de agentes anestésicos y tocolíticos, comparado con la cirugía fetoscópica[3].

Tratatamiento intraparto Ex-Útero (EXIT): Constituye una variante de la operación cesárea. Es realizado próximo al término de la gestación en fetos que se prevé dificultad en el manejo de la vía aérea al momento del nacimiento. La sospecha se basa en el diagnóstico antenatal mediante ecografía de masas cervicales o laríngeas, obstrucción congénita de la vía aérea alta o micrognatia severa. La vía aérea del feto se puede asegurar mientras éste aún recibe oxigenación desde la placenta, o bien puede ser conectado a membrana de oxigenación extracorpórea (ECMO) para garantizar la oxigenación, previo al clampeo del cordón umbilical[4].

\section{Riesgo materno y fetal}

La indicación de realizar una cirugía fetal debe ser cuidadosamente evaluada por un equipo multidisciplinario, considerando que el procedimiento no provee beneficio directo a la madre, por lo que se debe sopesar el riesgo materno versus el beneficio al feto. Por lo tanto, el procedimiento sólo debe ser considerado si el riesgo de muerte o secuelas neonatales son mayores si la patología es intervenida posterior al nacimiento en vez de en la vida intrauterina. Concomitantemente, el riesgo de complicaciones maternas debe ser aceptablemente bajo.

Las complicaciones intraoperatorias más frecuentes son hipotensión y sangrado, las que ocurren con mayor frecuencia en la cirugía abierta. En cuanto a las complicaciones postoperatorias, las más frecuentes son el parto prematuro, ruptura prematura de membranas, y edema pulmonar en la madre por sobrecarga de volumen hasta en un $10 \%$ de los casos, por lo que la fluidoterapia en estas pacientes debe ser cuida-

\section{Tabla 1. Procedimientos mínimamente invasivos}

(Adaptado de Hoagland y Chatterjee, 2017)

\begin{tabular}{ll}
\hline Procedimientos con aguja guiados por ultrasonido & $\begin{array}{l}\text { Toma de muestra percutánea de cordón umbilical, ablación por radio- } \\
\text { frecuencia de gemelo no viable, valvuloplastía con balón }\end{array}$ \\
Intervenciones fetoscópicas & $\begin{array}{l}\text { Fotocoagulación láser en transfusión feto-fetal, coagulación de cordón } \\
\text { umbilical, liberación de banda amniótica, ablación de válvulas uretrales } \\
\text { posteriores }\end{array}$ \\
\hline
\end{tabular}


dosa[3]. Por lo anteriormente mencionado, el periodo postoperatorio requiere estrecha vigilancia materna y fetal.

\section{Consideraciones anestésicas}

Los objetivos de la anestesia en cirugía fetal son:

- Mantener la seguridad materna durante el procedimiento y minimizar el riesgo perioperatorio.

- Asegurar la oxigenación fetal mediante la mantención de una adecuada circulación útero-placentaria.

- Proveer las condiciones quirúrgicas adecuadas necesarias para el tipo de intervención, particularmente el tono uterino e inmovilidad fetal.

- Mantener la homeostasis fetal mediante adecuada analgesia.

- Limitar la administración de fluidos para evitar los efectos adversos de la fluidoterapia.

- Desarrollar un plan de analgesia postoperatoria.

- Eventual necesidad de manejo tocolítico en el postoperatorio.

\section{Manejo perioperatorio}

\section{Evaluación preoperatoria}

La evaluación debe ser realizada por un equipo multidisciplinario de especialistas incluyendo cirujanos fetales, ginecólogos, anestesiólogos y neonatólogos. Se debe asegurar la disponibilidad de equipamiento y personal entrenado ante eventual parto pretérmino y cirugía fetal de emergencia.

El anestesiólogo debe conocer de manera detallada las comorbilidades maternas y complicaciones obstétricas previas. Cualquier enfermedad cardiaca o pulmonar significativa, o condición que implique riesgo anestésico aumentado para la madre constituye contraindicación para la cirugía fetal. En cuanto al feto, es importante para el anestesiólogo conocer la frecuencia cardiaca fetal basal, función cardiaca fetal evaluada mediante ecocardiografía, peso fetal estimado para dosificación de drogas y localización de la placenta.

Es importante señalar que debe contarse además con disponibilidad de sangre O-negativa, citomegalovirus-negativa y leucorreducida $(5-10 \mathrm{ml} / \mathrm{kg}$ ) en caso de ser necesario transfundir hemoderivados al feto[4].

\section{Anestesia, analgesia y reanimación fetal}

Los fetos en segundo y tercer trimestre de gesta- ción poseen el desarrollo neurológico suficiente para desarrollar una respuesta nociceptiva ante un estímulo doloroso[5], por lo tanto, la analgesia fetal no sólo constituye un fin en sí mismo, sino que también es una herramienta para inhibir movimientos del feto durante el procedimiento, lograr relajación uterina y disminuir la respuesta fetal al estrés, la cual puede contribuir al parto pretérmino. Por lo tanto, se debe proveer una analgesia adecuada en procedimientos potencialmente dolorosos para el feto.

Las vías de administración de drogas al feto son la intramuscular, a través de los vasos umbilicales, intramniótica, y transplacentaria mediante la administración materna de drogas intravenosas que atraviesen la barrera placentaria. La vía más utilizada es la intramuscular. Las drogas deben ser preparadas mediante técnica estéril y estar disponibles en el campo quirúrgico para administrarlas al feto. La posología de drogas fetales vía intramuscular se resumen en la Tabla 2.

\section{Técnica anestésica}

Procedimientos mínimamente invasivos: Son realizados habitualmente bajo anestesia local o neuroaxial. Estas técnicas anestésicas no proveen analgesia ni inmovilidad fetal[6]. La administración de remifentanil en infusión continua $(0,1-0,2 \mathrm{mcg} / \mathrm{kg} / \mathrm{min})$ ha demostrado proveer excelente sedación materna e inmovilización fetal, sin episodios de apnea en la madre y con parámetros hemodinámicos maternos y fetales estables[7]. La utilización de técnicas neuroaxiales asociadas a remifentanil en cirugía fetoscópica proporciona adecuadas condiciones quirúrgicas con menores complicaciones anestésicas. Se sugiere administrar atropina previo al inicio de infusión de remifentanil para prevenir bradicardia fetal. En caso de no lograr inmovilidad fetal con esta técnica, se recomienda administrar fentanilo, asociado a un relajante neuromuscular y atropina vía intramuscular fetal.

Los estándares de monitorización de la ASA son suficientes para controlar a la madre en cirugía fetos-

Tabla 2. Dosificación de drogas para administración
fetal IM


Tabla 3. Comparación del manejo de distintos tipos de cirugía fetal.

(Adaptado de Sviggum, 2013)

\begin{tabular}{|c|c|c|c|}
\hline & Cirugía Abierta & Fetoscopía & EXIT \\
\hline Edad gestacional & $\begin{array}{l}\text { Segundo semestre tardío/ } \\
\text { tercer trimestre temprano }\end{array}$ & $\begin{array}{l}\text { Segundo semestre tardío/ } \\
\text { tercer trimestre temprano }\end{array}$ & Término de la gestación \\
\hline Anestesia materna & $\begin{array}{l}\text { General, epidural para anal- } \\
\text { gesia postoperatoria }\end{array}$ & Local o neuroaxial \pm sedación & $\begin{array}{l}\text { General } \pm \text { epidural para anal- } \\
\text { gesia postoperatoria }\end{array}$ \\
\hline Tono uterino deseado & Relajación completa & Relajación mínima & Relajación completa \\
\hline Anestesia fetal & $\begin{array}{l}\text { Agentes inhalatorios trans- } \\
\text { placentarios. Opioides o } \\
\text { bloqueador neuromuscular } \\
\text { directo (IM) }\end{array}$ & $\begin{array}{l}\text { Opioides y bloqueador neu- } \\
\text { romuscular directo (IM) u } \\
\text { opioide transplacentario }\end{array}$ & $\begin{array}{l}\text { Agentes inhalatorios trans- } \\
\text { placentarios, opioides o } \\
\text { bloqueador neuromuscular } \\
\text { directo (IM) }\end{array}$ \\
\hline Riesgo de parto prematuro & Incrementado & Mínimo & No aplica \\
\hline $\begin{array}{l}\text { Monitorización invasiva de } \\
\text { presión arterial }\end{array}$ & Sí & No & Sí \\
\hline
\end{tabular}

cópica. La frecuencia cardiaca fetal debe ser monitorizada al principio y al final del procedimiento.

Cirugía fetal abierta: Se realizan bajo anestesia general. Previo a la inducción, se instala un catéter peridural, dado que la analgesia materna postoperatoria constituye un factor fundamental de la prevención del parto pretérmino. La analgesia peridural debe ser iniciada al término del procedimiento, considerando el riesgo de hipotensión intraoperatoria.

Además de los estándares de monitorización ASA, se debe instalar línea arterial para estrecha vigilancia hemodinámica. Usualmente no se requiere acceso venoso central. Se sugiere limitar la administración de volumen y privilegiar la administración de drogas vasoactivas en caso de hipotensión, dado el riesgo de edema pulmonar postoperatorio[8]. La monitorización fetal, una vez hecha la histerotomía, puede ser realizada con oxímetro de pulso, ecocardiografía fetal, electrodos fetales en el cráneo y muestra de sangre del cordón umbilical. La analgesia e inmovilidad fetal se logran a través de la administración intramuscular de fentanil y un bloqueador neuromuscular no despolarizante.

La evidencia disponible a la fecha sugiere que mantención de la anestesia se realice con anestésicos volátiles, alcanzando entre 2 a 3 veces la concentración alveolar mínima al momento de la histerotomía para lograr relajación uterina[1],[2],[4]. Sin embargo, la FDA publicó recientemente advertencias sobre posible daño neurocognitivo posterior a la exposición a la anestesia general en neonatos y fetos[9]. Estudios preclínicos en animales y primates no humanos han demostrado apoptosis neuronal y supresión de la neurogénesis hipocampal en ratas recién nacidas tras la administración de agonistas GABA, como anestésicos inhalatorios (isoflurano, sevoflurano, desflurano), benzodiacepinas y propofol[10],[11],[12]. También se han visto implicados antagonistas de receptores NMDA, como ketamina[13].

Un estudio poblacional notó un incremento en la incidencia de autismo en los niños que recibieron anestesia general al momento de la cesárea en comparación a aquellos niños que nacieron por parto vaginal o cesárea bajo anestesia neuroaxial[14]. Sin embargo, estudios clínicos en niños durante la infancia temprana han mostrado que exposiciones únicas y de corta duración a anestesia general no se han relacionado con deterioro cognitivo[15].

Dada la preocupación del efecto de los anestésicos generales en la sinaptogénesis del feto, la FDA recomienda utilizar para sedación en embarazadas anestésicos no relacionados con receptor GABA tales como remifentanil y dexmedetomidina, minimizar la duración de la exposición a anestésicos inhalatorios (idealmente menor a 3 horas), y minimizar los tiempos entre el inicio de la anestesia y el de la cirugía. Para lograr la relajación uterina deseada, se sugiere evitar aumentar la concentración alveolar mínima de los anestésicos inhalatorios, y en vez de ello complementar la tocólisis intraoperatoria con sulfato de magnesio, atosiban o nitroglicerina[9].

Una vez realizada la histerorrafia, se puede realizar traslape a anestesia total intravenosa (TIVA) para disminuir los efectos de los anestésicos volátiles en el tono uterino[4]. La extubación debe ser cuidadosa, asegurando reversión completa del bloqueo neuromuscular y que la paciente se encuentre completamente despierta y responda a órdenes simples. Dado 
la manipulación uterina, se debe mantener tocólisis al menos en las primeras 48 horas posteriores al procedimiento. De preferencia se utiliza sulfato de magnesio en infusión continua.

Tratatamiento intraparto Ex-Útero (EXIT): El manejo inicial es similar al de la cirugía fetal abierta. Se instala un catéter peridural y posteriormente se realiza inducción de anestesia general. También se debe instalar línea arterial para monitorización hemodinámica y vías venosas periféricas de grueso calibre.

Al momento de la histerotomía se complementan los anestésicos inhalatorios con tocolíticos intravenosos. A diferencia de la cirugía fetal abierta, no se requiere de manejo tocolítico posoperatorio y se puede administrar fluidoterapia en caso de hipotensión, dado que existe bajo riesgo de edema pulmonar. Posterior al alumbramiento se administran agentes uterotónicos para disminuir la incidencia de hemorragia posparto.

Para asegurar la vía aérea fetal debe estar presente un anestesiólogo encargado del feto, y un otorrinolaringólogo en caso de fracaso de intubación y necesidad de acceso infraglótico.

Luego de la histerotomía, se exterioriza parcialmente el feto y se aborda la vía aérea mediante instrumental estéril previamente preparada en el campo quirúrgico. Una vez intubado el feto, se debe corroborar la posición del tubo con fibrobroncoscopio. Los pulmones no deben ser ventilados hasta justo antes del clampeo de cordón umbilical, para garantizar una adecuada transición de la circulación fetal a la neonatal. Finalizado el procedimiento, se realiza la extubación materna y la paciente se traslada a la Unidad de Recuperación Postanestésica para continuar los cuidados postoperatorios.

\section{Descripción de casos}

Desde abril del año 2015 a marzo de 2019, sesenta y tres pacientes se sometieron a cirugía por indicación fetal. En 1 embarazo gemelar se realizaron 2 cirugías durante la gestación. En total fueron 64 procedimientos. La edad promedio materna fue de 28,5 \pm 6 años. La edad gestacional promedio fue 19,7 semanas \pm 3 días. Todas las cirugías fueron realizadas durante el segundo trimestre de embarazo.

En cuanto al tipo de gestación, 59 cirugías fueron realizadas en embarazos múltiples, de éstos 55 en embarazos gemelares dobles monocoriales biamnióticos y 3 embarazos gemelares triples. En los embarazos gemelares, 54 cirugías fueron por síndrome de transfusión feto-fetal y 5 por secuencia TRAP.

Las 5 pacientes intervenidas por otras causas correspondían a embarazos únicos cuya indicación quirúrgica fue una malformación fetal. Entre ellas se registró brida amniótica, megavejiga y malformación adenomatoídea quística pulmonar.

Todos los procedimientos fueron realizados mediante fetoscopía. No hubo intervenciones fetales con técnica abierta ni procedimientos EXIT.

Sobre la técnica anestésica, se realizaron 2 cirugías con anestesia general, 42 con anestesia espinal y 20 con anestesia espinal-epidural combinada. Se utilizó monitorización estándar en 62 procedimientos y en 2 se agregó línea arterial. En 42 procedimientos se utilizó remifentanil TCI para sedación materna e inmovilización fetal durante la anestesia neuroaxial.

De las complicaciones maternas perioperatorias, en 1 procedimiento fetoscópico con anestesia neuroaxial por STFF, al día siguiente la madre presentó hemoperitoneo que requirió laparotomía exploradora de urgencia y, posteriormente, presentó insuficiencia respiratoria que requirió ventilación mecánica no invasiva. En una fetoscopía durante coagulación de comunicaciones arteriovenosas se produjo sangrado profuso que impidió terminar el procedimiento y posteriormente se desencadenó parto prematuro. Una madre requirió parche de sangre por cefalea post punción dural. Otra madre presentó sepsis de foco urinario en el puerperio.

Respecto a la evolución de los embarazos, en la ficha clínica se registró óbito de uno o ambos gemelos en 17 embarazos múltiples. Se produjeron 4 roturas prematuras de membranas (RPM), y en 1 amenaza de parto prematuro.

En la experiencia de nuestro centro el procedimiento más frecuente fue la fetoscopía en STFF, y la técnica anestésica más utilizada fue anestesia neuroaxial más sedación con remifentanil y monitorización estándar, lo que concuerda con la evidencia internacional. En la Tabla 4 se resumen las características de los procedimientos realizados en nuestro centro.

\section{Conclusiones}

El avance en técnicas quirúrgicas que permiten intervenir anomalías fetales ha posibilitado cambiar el pronóstico de ciertas enfermedades susceptibles de tratamiento en la vida intrauterina. Dadas recientes investigaciones referentes al efecto de la anestesia en el neurodesarrollo fetal, se han precisado de nuevas técnicas para proteger al feto de los efectos deletéreos de determinados agentes anestésicos.

Futuros estudios de seguimiento están siendo desarrollados en nuestro centro hospitalario para poder 


\begin{tabular}{llc}
\hline & Tabla 4. Resumen de intervenciones en nuestro centro & \\
\hline & & Porcentaje (n) \\
Tipo de embarazo & Único & $7,9 \%(5)$ \\
& Gemelar doble & $87,3 \%(55)$ \\
& Gemelar triple & $4,8 \%(3)$ \\
Indicación quirúrgica & STFF & $84,4 \%(54)$ \\
& Secuencia TRAP & $7,8 \%(5)$ \\
Tipo de anestesia & Malformación fetal & $7,8 \%(5)$ \\
& General & $3,1 \%(2)$ \\
Complicaciones fetales & Espinal & $65,6 \%(42)$ \\
& Neuroaxial + sedación & $31,3 \%(20)$ \\
& Óbito fetal & $65,6 \%(42)$ \\
\hline
\end{tabular}

determinar desenlaces materno-fetales a mediano y largo plazo.

Queremos manifestar nuestro agradecimiento al
Servicio de Ginecología y Obstetricia del Hospital Clínico San Borja-Arriarán, por su apoyo en el acceso a la información y seguimiento de estas pacientes.

\section{Referencias}

1. Hoagland M, Chatterjee D. Anesthesia for fetal surgery. Pediatric Anesthesia ISSN 11555645, 2016.

2. Brusseau R, Mizrahi-Arnaud A. Fetal anesthesia and pain management for intrauterine therapy. Clin Perinatol. 2013 Sep;40(3):429-42. https://doi. org/10.1016/j.clp.2013.05.006 PMID:23972749

3. Manrique S, Maiz N, García I, Pascual M, Perera R, Arévalo

$S$, et al. Maternal anaesthesia in open and fetoscopic surgery of foetal open spinal neural tube defects: A retrospective cohort study. Eur J Anaesthesiol. 2019 Mar;36(3):175-84. https://doi.org/10.1097/ EJA.0000000000000930 PMID:30507621

4. Sviggum HP, Kodali BS. Maternal anesthesia for fetal surgery. Clin Perinatol. 2013 Sep;40(3):413-27. https://doi. org/10.1016/j.clp.2013.05.012 PMID:23972748

5. Fisk NM, Gitau R, Teixeira JM, Giannakoulopoulos X, Cameron $A D$, Glover VA. Effect of direct fetal opioid analgesia on fetal hormonal and hemodynamic stress response to intrauterine needling. Anesthesiology. 2001 Oct;95(4):828-35. https:// doi.org/10.1097/00000542200110000-00008 PMID:11605920

6. Van de Velde M, De Buck F, Van Mieghem T, Gucciardo L, De Koninck P, Deprest J. Fetal anaesthesia: is this necessary for fetoscopic therapy? Fetal Matern Med Rev. 2010;21(1):2435. https://doi.org/10.1017/ s0965539509990179.

7. Missant C, Van Schoubroeck D, Deprest J, Devlieger R, Teunkens $A$, Van de Velde $M$.
Remifentanil for foetal immobilisation and maternal sedation during endoscopic treatment of twin-to-twin transfusion syndrome : a preliminary dosefinding study. Acta Anaesthesiol Belg. 2004;55(3):239-44. PMID:15515301

8. Golombeck K, Ball RH, Lee $H$, Farrell JA, Farmer DL, Jacobs $V R$, et al. Maternal morbidity after maternal-fetal surgery. Am J Obstet Gynecol. 2006 Mar;194(3):834-9. https://doi. org/10.1016/j.ajog.2005.10.807 PMID:16522421

9. Olutoye OA, Baker BW, Belfort MA, Olutoye OO. Food and Drug Administration warning on anesthesia and brain development: implications for obstetric and fetal surgery. Am J Obstet Gynecol. 2018 Jan;218(1):98-102. https://doi. org/10.1016/j.ajog.2017.08.107 PMID:28888583 
10. Creeley CE, Dikranian KT, Dissen GA, Back SA, Olney JW, Brambrink AM. Isoflurane-induced apoptosis of neurons and oligodendrocytes in the fetal rhesus macaque brain. Anesthesiology. 2014 Mar;120(3):62638. https://doi.org/10.1097/ ALN.0000000000000037 PMID:24158051

11. Creeley $C$, Dikranian $K$, Dissen G, Martin L, Olney J, Brambrink A. Propofol-induced apoptosis of neurones and oligodendrocytes in fetal and neonatal rhesus macaque brain. Br J Anaesth. 2013 Jun;110 Suppl 1:i29-38. https:// doi.org/10.1093/bja/aet173 PMID:23722059

12. Shao C-Z, Xia K-P. Sevoflurane anesthesia represses neurogenesis of hippocampus neural stem cells via regulating microRNA183-mediated NR4A2 in newborn rats. Journal of Cellular Physiology. Wiley; 2018 Sep 7;234(4):3864-73. Available from: http://dx.doi.org/10.1002/ jcp. 27158

13. Young $C$, Jevtovic-Todorovic V, Qin YQ, Tenkova T, Wang $\mathrm{H}$, Labruyere J, et al. Potential of ketamine and midazolam, individually or in combination, to induce apoptotic neurodegeneration in the infant mouse brain. Br J Pharmacol. 2005 Sep;146(2):189-97. https://doi. org/10.1038/sj.bjp.0706301 PMID:15997239
14. Chien LN, Lin HC, Shao YH, Chiou ST, Chiou HY. Risk of autism associated with general anesthesia during cesarean delivery: a populationbased birth-cohort analysis. J Autism Dev Disord. 2015 Apr;45(4):932-42. https://doi. org/10.1007/s10803-0142247-y PMID:25256350

15. Sun LS, Li G, Miller TL, Salorio C, Byrne MW, Bellinger DC, et al. Association between a single general anesthesia exposure before age 36 months and neurocognitive outcomes in later childhood. JAMA. 2016 Jun;315(21):2312-20. https://doi. org/10.1001/jama.2016.6967 PMID:27272582 